\title{
Notas sobre flora vascular de la provincia de Toledo (Península Ibérica, España)
}

Gonzalo Hernández Palacios

Área de Botánica. Instituto de Ciencias Ambientales, Universidad de Castilla-La Mancha. 45071 Toledo.

\section{Resumen}

\section{Correspondencia}

G. Hernández-Palacios

E-mail: gonzahp@gmail.com

Recibido: 30 enero 2013

Aceptado: 26 marzo 2013

Publicado on-line: 17 abril 2013

\begin{abstract}
Se señalan y comentan 60 taxones de plantas vasculares recolectadas en diferentes unidades corológicas de la provincia de Toledo, de las cuales nueve son nuevas en la misma. En el conjunto restante, se amplia el conocimiento sobre su corología o bien se añade la presencia en Toledo a la distribución provincial del proyecto Flora Iberica.
\end{abstract}

Palabras clave: Plantas vasculares, Flora, Corología, Península Ibérica, Castilla-La Mancha, Toledo.

\begin{abstract}
Notes on the vascular flora of Toledo province (Iberian Peninsula, Spain)

This paper presents short comments about the occurrence of 66 taxa recorded on different chorological units belonging to Toledo province, nine of them are remarked for first time in this area. For the remaining group its chorological knowledge is enhanced, or its presence is added to distribution data provided on Flora Iberica Project.
\end{abstract}

Key words: Vascular plants, Flora, Chorology, Iberian Peninsula, Castilla-La Mancha, Toledo.

\section{Introducción}

El estudio de la diversidad vegetal de Toledo conoció un desarrollo importante en las decadas finales del siglo pasado con los diferentes estudios iniciados sobre todo en la Universidad Complutense de Madrid sobre territorios más o menos amplios de esta provincia, cuyo cuerpo florístico quedaba de esta forma cerrado o conocido en unos niveles satisfactorios, aunque no consolidado en una síntesis de consulta. Este aspecto vino a solucionarse con la publicación de una lista bibliográfica exhaustiva en años ya más recientes (Sanz Elorza 2006), a la que han seguido otras aportaciones, junto con la publicación de la síntesis de comunidades vegetales reconocidas hasta hoy (Fernández González et. al 2012).

El establecimiento de un grupo de investiga- ción en la ciudad de Toledo, adscrito a la universidad regional, ha supuesto un impulso definitivo al estudio de la botánica en el territorio considerado y sus provincias vecinas, cuya riqueza específica pretende testimoniar el herbario CALM del Instituto de Ciencias Ambientales de esta universidad. Se trata de una colección joven, de reciente creación, pero cuyos fondos crecen rápidamente y con una informatización de especímenes completa desde sus primeros pliegos.

Sin embargo, de la misma forma que sucede en otras provincias, existen grupos de plantas insuficientemente conocidos o zonas poco visitadas, razón por la que presentamos esta lista de notas corológicas con los hallazgos de nuestras campañas llevadas a cabo estos últimos años en Toledo.

En la confección de este trabajo se ha consultado la información de máxima utilidad que ofrece 
el programa ANTHOS 2011 (www.anthos.es) como fuente primaria de información bibliográfica y representación visual de áreas de distribución de especies en la Península Ibérica, aunque posteriormente hemos obtenido los datos en el texto de las publicaciones que refiere el programa.

Androsace elongata var. breistrofferi Charpin \& Greuter

Pulgar, cerro del Gango, pastos anuales sobre migmatitas, 770 msnm, 30SVJ0096, 14-IV-2001, Hernández Palacios s.n. (CALM-2936).

Interesante nanoterófito que se extiende por los ambientes esteparios del valle del Ebro y del Duero, pero que resulta mucho más raro en la cuenca del Tajo, donde sólo conocemos la localidad toledana indicada por Segura Zubizarreta (1975) en Mora; nosotros la hemos herborizado también en la Meseta Cristalina de Toledo, en comunidades silicibasófilas de terófitos sobre suelos pedregosos. Reconocemos las dificultades de herborización en taxones de tan pequeño tamaño, así como la brevedad y precocidad de su floración, que se han propuesto en ocasiones para explicar la escasez de referencias (Molero \& Montserrat 1983), pero al menos en lo referente a la provincia de Toledo y la cuenca del Tajo creemos que es una planta francamente escasa.

Anthyllis vulneraria subsp. gandogeri (Sagorski) W. Becker

Urda, encinares, 950 msnm, 30SVJ4155, 24-V2008, Hernández Palacios s.n. (CALM-2935).

Poco conocida en la provincia, sólo encontramos una mención genérica de Velasco (1978) en los Montes de Toledo, y otra reciente de Baonza et al. (2010) en el Macizo de las Guadalerzas. Asimismo hemos visto material de herbario procedente de la llanura manchega, en el extremo oriental de la provincia (MA-64909; MA-64974).

Astragalus incanus L. subsp. incanus

Yepes, salviares en calizas pontienses, $600 \mathrm{msnm}$, 30SVK3821, 5-V-2009, Hernández Palacios s.n. (CALM-2932); Villatobas, salviares en calizas pontienses, $750 \mathrm{msnm}$, 30SVK8012, 4-VII-2000, Hernández Palacios s.n. (CALM-3047).

Iberonorteafricana de área amplia, aunque mucho más escasa que la subespecie nummularioides (Desf.) Maire, con la que comparte el territorio oriental de la provincia. Podlech (2000) ya indica- ba su presencia en la provincia, pero tan sólo conocemos la mención previa de Rivas Goday \& Rivas Martínez (1969), sub A. incurvus Desf. No obstante encontramos otros testimonios (MA336679, MA-67543) también del Sector Manchego, donde según lo visto se comportaría como gipsófita preferente.

\section{Avellinia michelii (Savi) Parl.}

Navamorcuende, claros de matorral, $750 \mathrm{msnm}$, 30TUK5543, 11-V-2002, Hernández Palacios s.n. (CALM-1083); Urda, tomillares, $860 \mathrm{msnm}$, 30SVK4057, 24-V-2008, Hernández Palacios s.n. (CALM-2931).

Aportamos nuevas localidades de esta pequeña gramínea que recientemente indicaban como novedad Baonza et al. (2010) en el tramo oriental de los Montes de Toledo, por lo que su área provincial comprendería la alineación oretana y el bloque serrano de San Vicente, evitando los medios continentalizados y semiáridos de la fosa del Tajo.

Avena barbata subsp. castellana Romero Zarco

Argés, bordes de olivar, 720 msnm, 30SVK0905, 8-VI-2002, Hernández Palacios s.n. (CALM2930).

Taxón un tanto ignorado en trabajos locales. Nuestra localidad es la segunda en la provincia de Toledo (Romero Zarco 1990). Revisando material perteneciente a las subespecies afines, hemos encontrado muestras incluibles en este taxón tanto en la cuenca miocena del Tajo (MAF-117131, Borox) como en el macizo de las Villuercas (MAF77922), no obstante remarcamos la dificultad de observar satisfactoriamente las lodículas del material prensado, que junto con la inserción de la flor, constituyen los caracteres diagnósticos. En el caso de este último hemos encontrado una mayor constancia de formas estrechas en el callo, junto con arístulas menores de $5 \mathrm{~mm}$, mientras que en las lodículas, como señala recientemente Romero Zarco (2009), puede o no reconocerse un diente lateral.

Avena barbata subsp. lusitanica (Tab. Morais) Romero Zarco

Caleruela, comunidades de Lavandula stoechas, 450 msnm, 30SUK0816, 5-V-2008, Hernández Palacios s.n. (CALM-2928); Pulgar, bordes de cultivos, 730 msnm, 30SVJ0191, 14-IV-2001, Hernández Palacios s.n. (CALM-2929). 
Es el taxón más extendido en el Sector ToledanoTagano de la provincia de Toledo dentro del complejo A. barbata Pott., pero las referencias expresas son muy escasas (Baonza et al. 2010).

\section{Avena eriantha Durieu}

Dos Barrios, Arroyo del Valle, $650 \mathrm{msnm}$, 30SVK5814, 30-IV-2000, Hernández Palacios s.n. (CALM-954); Yepes, salviares, $600 \mathrm{msnm}$, 30SVK3821, 5-V-2009, Hernández Palacios s.n. (CALM-2925); Santa Cruz de la Zarza, aljezares, 690 msnm, 30SVK8229, 30-IV-2010, Hernández Palacios s.n. (CALM-2926).

Especie subgipsófita y con una interesante distribución de tipología iranoturaniana. Añadimos aquí la segunda y posteriores citas en Toledo, provincia en la que no se había vuelto a encontrar desde una primera recolección en 1968 (Montserrat \& Cardona 1983); ampliamos así su distribución mediante estas localidades, las más meridionales de la cuenca del Tajo. El comportamiento observado la sitúa en el seno comunidades anuales o fruticosas de especies subnitrófilas, siempre formando parte del mosaico cenológico de los paisajes yesíferos miocenos, donde no resulta rara.

\section{Avenula bromoides subsp. pauneroi Romero Zarco}

Villatobas, salviares en claros de encinar, 750 msnm, 30SVK8012, 7-IV-2000, Hernández Palacios s.n. (CALM-2924).

Común en las formaciones fruticosas del Sector Manchego, tanto en yesos como en calizas e incluso migmatitas del límite oriental de la Meseta Cristalina. Sanz Elorza (2006) no recogía este taxón en su síntesis de la flora provincial, y de hecho sólo conocemos dos referencias de Romero Zarco (1984) a partir de material de herbario recolectado en este sector corológico.

\section{Avenula marginata subsp. albinervis (Boiss.)} Romero Zarco

Navamorcuende, Las Cruces, tomillares en litosuelos, 1300 msnm, 11-V-2002, Hernández Palacios s.n. (CALM-929).

Adoptamos el criterio taxonómico de Romero Zarco (1993). En el conjunto de taxones afines a Avenula marginata (Love) J. Holub, s.l. (A. sulcata Gay ex Boiss.), se trataría del menos extendido en la provincia, ya que hasta ahora únicamente se había reconocido en el límite de los Montes de
Toledo con la llanura manchega (Marcos 1985). Tiene preferencia por suelos poco evolucionados. Suponemos la existencia de otras poblaciones a lo largo de las sierras oretanas.

\section{Bupleurum baldense Turra}

Noblejas, salviares sobre calizas, $730 \mathrm{msnm}$, 30SVK6028, 27-V-2000, Hernández Palacios s.n. (CALM-937).

Pequeño terófito de distribución escasamente documentada en la provincia. Laorga (1986) la señalaba también, como especie rara, en la Mesa de Ocaña. Añadimos otra cuadrícula de $10 \mathrm{~km}$ de lado en su distribución provincial. A la vista de las serias dificutades para distinguir este taxón de su afín B. semicompositum L. que expone Neves (2003), sería conveniente revisar más adelante la identidad de las citas oretanas de B. baldense (Navarro 1996, Baonza et al. 2010).

Bupleurum rigidum L. subsp. paniculatum (Brot.) H. Wolff. in Engl.

Urda, encinares incipientes, $1030 \mathrm{msnm}$, 30SVJ4257, 24-V-2008, Hernández Palacios s.n. (CALM-2923).

Taxón iberonorteafricano que sustituye a $B$. rigidum L. subsp. rigidum en el occidente peninsular y sobre suelos oligótrofos. Presente a lo largo de las elevaciones oretanas, especialmente en las centrales y más orientales (Baonza et al. 2010).

\section{Calamintha nepeta (L.) Savi subsp. nepeta}

Mohedas de la Jara, umbría de la Sierra de Altamira, melojares en cuarcitas, $1000 \mathrm{msnm}$, 30SUJ1679, 8-VI-2003, Hernández Palacios s.n. (CALM-2922).

Otra localidad para el contacto de los Montes de Toledo con el Macizo de las Villuercas, donde había sido recolectada anteriormente (Ladero et al. 1985). Junto con el bloque de la Sierra de San Vicente (Cantó 2004), constituyen las dos únicas estaciones conocidas en la provincia de Toledo. Indicada de forma dudosa por Morales (2010) debido a la escasez de referencias y recolecciones respaldadas en herbarios (Morales 1997).

Callipeltis cucullaris (L.) Steven

Dosbarrios, Barranco de la Cañada de la Mora, atochares, $680 \mathrm{msnm}, 30 \mathrm{SVK} 5615$, 30-IV-2000. Hernández Palacios s.n. (CALM-981).

Planta escasa que ya recolectara Boissier (1838) 
en la localidad madrileña de Aranjuez y mucho más tarde Laorga (1986), por lo que añadimos ahora una primera cita en Toledo. Constatamos asimismo su extrema rareza en las localidades visitadas. Se trata por lo tanto de uno de los taxones más escasos en la cuenca miocena del Tajo, prueba de lo cual es el dilatado lapso de años que separa la primera y segunda cita en Aranjuez, una de las localidades mejor estudiadas botánicamente de la Península Ibérica.

\section{Chamaesyce nutans (Lag.) Small}

*Toledo, Vega Baja, jardines abandonados, 460 msnm, 30SVK1013, 17-IX-2001, Hernández Palacios s.n. (CALM-2921).

Euforbiácea de origen neotropical, que hemos localizado en jardines semiabandonados. Se trata de la primera recolección en Toledo.

\section{Chamaesyce prostrata (Aiton) Small}

Toledo, Vega Baja, jardines abandonados, 460 msnm, 30SVK1013, 17-IX-2001, Hernández Palacios s.n. (CALM-2920).

Segunda localidad provincial de esta especie (Baonza et al. 2010) también de origen neotropical, que convivía con la especie anterior en los mismos medios.

\section{Clypeola jonthlaspi subsp. microcarpa (Moris)} Arcang.

Dos Barrios, barranco del Arroyo de la Cañada de la Mora, pastizales en calizas, $680 \mathrm{msnm}$, 30SVK5615, 30-IV-2000, Hernández Palacios s.n. (CALM-946).

Este taxón ha sido admitido por diferentes autores con rango varietal, específico, o subespecífico. Dentro del complejo polimórfico de $C$. jonthlaspi L., las plantas con frutos pequeños e hirsutos únicamente en el disco seminal parecen ser las formas más constantes, y tal distribución del indumento no existiría en frutos de mayor tamaño (Chaytor \& Turril 1935). Añadimos que su número cromosómico $(2 \mathrm{n}=32)$ no ha sido hasta ahora encontrado en el complejo de la especie. Por estas razones preferimos reconocer valor taxonómico a esta subespecie. Es francamente rara en la Mesa e Ocaña, donde mantendría las únicas poblaciones toledanas. Su presencia en suelos yesíferos de la Sagra de Toledo fue dada a conocer por Izco (1974) aunque no hemos encontrado material de herbario para atestiguarlo.

\section{Eleocharis palustris subsp. vulgaris Walters}

Navalcán, bordes del embalse, $370 \mathrm{msnm}$, 30TUK1735, 17-IV-2000, Hernández Palacios s.n. (CALM-2918); Noez, Valdelamora, camino a Casasbuenas, juncales, $710 \mathrm{msnm}$, 30SVK0000, 21-IV-2001, Hernández Palacios s.n. (CALM2919).

Indicamos las primeras localidades provinciales de este alopoliploide de muy amplia distribución. Recolectada anteriormente en Talavera de la Reina y localidades cercanas (MA-652335, MA652340). La separación respecto a la subespecie típica no resulta fácil. Uno y otro taxón se observan en las localidades toledanas visitadas, por lo que creemos que deben tener lugar los fenómenos de hibridación que refieren Jiménez Mejías \& Luceño (2007).

\section{Erysimum lagascae Rivas Goday \& Bellot}

San Pablo de los Montes, melojares, $1160 \mathrm{msnm}$, 30SUJ8476, 22-V-2010, Hernández Palacios s.n. (CALM-2916).

No conocida hasta la fecha en los Montes de Toledo, en cambio resulta más frecuente en el bloque de la Sierra de San Vicente (Cantó 2004, García Mateo 2003). Endémica del centro-sur de la Península Ibérica.

\section{Euphorbia exigua subsp. merinoi M. Laínz}

Navalcán, bordes del embalse, majadales, 380 msnm, 30TUK1735, 17-IV-2000, Hernández Palacios s.n. (CALM-1011); Layos, junto al embalse de Guajaraz, 620 msnm, 30SVK0705, 5-V-2001, Hernández Palacios s.n. (CALM-2917).

Taxón iberonorteafricano (Larache y Fez, Marruecos) del que precisamos, con estas dos localidades (segunda y tercera en la provincia), su corología en Toledo. Representaría, a excepción de las poblaciones conquenses (Mateo \& Arán 2002), el límite oriental de su extensión. Cantó (2004) ya la indicó en la Sierra de San Vicente. Teniendo en cuenta su preferencia por suelos oligótrofos y climas de carácter atlántico, esperamos el hallazgo de futuras localidades en los Montes de Toledo.

\section{Euphorbia flavicoma DC. subsp. flavicoma}

Villatobas, salviares en litosuelos calizos, 700 msnm, 30SVK7111, Hernández Palacios s.n. (CALM-2915).

Se trata del taxón de mayor extensión geográfica y el que presenta un mayor grado de polimorfismo 
dentro de la especie, aunque es sumamente rara en el interior peninsular. Su presencia está documentada a lo largo de los Montes de Toledo, donde aparece muy puntualmente según la revisión que efectúa Simón i Pallisé (1993), pero es novedad en la cuenca miocena del Tajo. En la localidad visitada habita huecos de salviares muy ralos sobre suelos calcáreos pedregosos, lo que frente a las poblaciones oretanas (sobre cuarcitas y pizarras paleozoicas), concuerda más con la ecología conocida de este taxón.

Euphorbia helioscopia subsp. helioscopioides (Loscos \& J. Pardo) Nyman

Corral de Almaguer, salviares alterados, 680 msnm, 30SVK7106, 6-V-2010, Hernández Palacios s.n. (CALM-2914).

Primera localidad provincial de esta lechetrezna, escasa en la cuenca terciaria del Tajo. Convive con la subespecie típica, que resulta abundante en estas localidades según Laorga (1986) y el numeroso material de herbario que hemos visto (MAF109551; MAF-109548), si bien esta última habita en medios mucho más alterados. El pliego MA403359, recolectado en "Arroyo de la Cavina, entre la carretera de Andalucía y la de Toledo, 30SVJ4426", es localidad madrileña, lo que señalamos a efectos de hacer destacar su rareza en la región estudiada.

Euphorbia nevadensis subsp. nevadensis Boiss. \& Reut.

Caleruela, La Navarra, cantuesares en gravas, 450 msnm, 30SUK0816, 6-V-2008, Hernández Palacios s.n. (CALM-890).

Tercera cita en la provincia de Toledo (Cantó 1979, Arán 1995). Confirmamos así su presencia en la misma, que Benedí et al. (1997) consideran dudosa. Se distribuye por lo tanto de forma muy dispersa en el sector Toledano-Tagano.

Euphorbia sulcata Lens ex Loisel.

Dos Barrios, pastizales en calizas, $650 \mathrm{msnm}$, 30SVK5615, 30-IV-2000, Hernández Palacios s.n. (CALM-969); Corral de Almaguer, tomillares nitrófilos, $680 \mathrm{msnm}$, 30SVK7106, 6-V-2010, Hernández Palacios s.n. (CALM-3046).

Especie muy poco recolectada en la provincia, pero bastante común en el resto de la cuenca terciaria del Tajo, como señalaba Laorga (1986), quien aporta las únicas menciones previas que hemos podido encontrar en la bibliografía.

\section{Globularia vulgaris L.}

*Villatobas, romerales, $690 \mathrm{msnm}, 30$ SVK7110, 6-V-2010, Hernández Palacios s.n. (CALM2913).

Herborizada muy puntualmente en comunidades de Rosmarinus officinalis L. sobre suelos calizos esqueléticos en los que medra una exigua población. Se trata de la primera cita provincial. Las localidades más próximas son conquenses, situadas en el sur de la Sierra de Altomira. Destacamos la presencia en el centro y sur de la Mesa de Ocaña de otros taxones con similares requerimientos ecológicos y distribución, tales como Festuca hystrix Boiss. o Arctostaphyllos uva-ursi (L.) Spreng.

Helianthemum nummularium (L.) Mill.

*Ventas con Peña Aguilera, Puerto del Milagro, cultivos de cereal, $930 \mathrm{msnm}$, 30SUJ9277, 25-IV2010, Hernández Palacios s.n. (CALM-2909).

Novedad provincial. Se trata de una de las pocas especies ibéricas de este género con área de distribución principalmente septentrional y que se hace rara al sur del Sistema Central e Ibérico.

\section{Juncus bufonius L.}

Pulgar, Finca Alamedillas, 21-IV-2001, Hernández Palacios s.n. (CALM-2942).

Muy común en la mitad sur de la provincia de Toledo, pero omitido en la síntesis genérica de Romero Zarco (2010).

Jurinea humilis (Desf.) DC.

Villarrubia de Santiago, salviares, $740 \mathrm{msnm}$, 30SVK7026, 4-VII-2000, Hernández Palacios s.n. (CALM-2908).

Hemicriptófito de areal amplio pero poco conocido en la provincia de Toledo, donde solo existen algunas citas anteriores en las localidades próximas que indica Laorga (1986). Queda por lo tanto circunscrita a las comunidades fruticosas sobre suelos calizos que bordean la Mesa de Ocaña.

Lathyrus niger (L.) Bernh.

San Pablo de los Montes, melojares sobre cuarcitas, 1160 msnm, 30SUJ8476, 22-V-2010, Hernández Palacios s.n. (CALM-2907).

Añadimos una segunda referencia provincial de 
este elemento nemoral paleotemplado, poco conocido en los Montes de Toledo y que hemos herborizado en orlas de melojares supramediterráneos. Sólo conocemos la cita anterior de Velasco (1978) en el mismo sector corológico. Su distribución provincial se restringe al tramo central de la alineación oretana y la Sierra de San Vicente, de donde se conserva un testimonio en SALA.

\section{Launaea pumila (Cav.) O. Kuntze}

Noblejas, salviares alterados, en calizas, 730 msnm, 30SVK6126, 27-V-2000, Hernández Palacios s.n. (CALM-935); Huerta de Valdecarábanos, aljezares en yesos rojos, $650 \mathrm{msnm}, 30 \mathrm{SVK} 4814$, 17-V-2008, Hernández Palacios s.n. (CALM2906).

Iberonorteafricana subgipsófita y bastante rara en el Sector Manchego de Toledo, especialmente en los aljezares de la vertiente del Tajo. Únicamente encontramos las citas previas de Laorga (1986) en localidades próximas de la cuenca del Guadiana (Tembleque y La Guardia).

Leucanthemopsis pallida subsp. flaveola (Hoff. \& Link) Ladero \& Velasco

San Pablo de los Montes, melojares sobre cuarcitas, 1160 msnm, 30SUJ8476, 22-V-2010, Hernández Palacios s.n. (CALM-2944).

Hemicriptófito endémico de la mitad peninsular occidental con exigencias ecológicas similares a Lathyrus niger, y que señala el carácter atlántico de los melojares supramediterráneos oretanos. Las referencias en los Montes de Toledo son escasas en comparación con la abundancia relativa de las mariánicas (Martín-Blanco \& Carrasco 2005). Presente asimismo en la Sierra de San Vicente (Cantó 2004).

Lolium hybridum Hausskn.

* Guadamur, prados nitrificados, Arroyo de la Dehesa, $600 \mathrm{msnm}$, 30SVK0008, 28-IV-2001, Hernández Palacios s.n. (CALM-2954).

Especie de origen híbrido reconocible por sus glumas aristadas. Aportamos esta primera localidad en la provincia de Toledo.

\section{Malva trifida Cav.}

Yepes, pastos anuales sobre calizas pontienses, 610 msnm, 30SVK3831, 5-V-2009, Hernández Palacios s.n. (CALM-2904).
Terófito endémico de la mitad oriental de la Península Ibérica, no indicado para Toledo por Nogueira \& Paiva (1996) a pesar de la acumulación de numerosas citas anteriores en el tramo central de la cuenca del Tajo (Laorga 1986, Velasco 1978) y de algunos testimonios de herbario (MAF105575, MAF-110203).

Medicago arabica (L.) Huds.

Hinojosa de los Montes, Sierra de San Vicente, encinares, $640 \mathrm{msnm}$, 30TUK5341, 11-V-2002, Hernández Palacios s.n. (CALM-2903); Cobisa, pastos anuales en arcosas, $880 \mathrm{msnm}$, 30SVK1008, 5-V-2001, Hernández Palacios s.n. (CALM-2901).

Taxón de área muy amplia que aparece de forma dispersa en la mitad occidental de la provincia (Laorga, 1986; Cantó 2004, Baonza et al. 2010), pero no reconocido en la misma por Sales \& Hedge (1999).

\section{Medicago littoralis Rohde}

Yepes, olivares sobre calizas, $580 \mathrm{msnm}$, 30SVK3821, 5-V-2009, Hernández Palacios s.n. (CALM-2900).

Aportamos una nueva localidad de este terófito raro en el territorio provincial, donde había sido citado antes por Cantó (1979) de la Sierra de San Vicente y Laorga (1986) de la fosa del Tajo. Asimismo omitida por Sales \& Hedge (1994) en la provincia.

\section{Medicago truncatula Gaertn.}

Layos, Sierra de Layos, matorral de Cistus albidus, 770 msnm, 30SVJ1198, 1-VI-2001, Hernández Palacios s.n. (CALM-1071).

Completamos con esta especie los taxones de la sección Spirocarpos Ser. de este género. Resulta algo más extendido que la especie anterior pero igualmente mal conocido en la provincia. Las referencias más cercanas la sitúan en la cuenca yesífera miocena de Madrid (Rivas Goday \& al. 1959) y en el piedemonte septentrional de los Montes de Toledo (Castillo \& Carrasco 1992). Debe añadirse la provincia de Toledo a la distribución peninsular de Sales \& Hedge (1999).

Myosotis discolor subsp. dubia (Arrondeau) Blaise

Navalcán, bordes del embalse, 30TUK1735, 380 
msnm, 17-IV-2000, Bernal, Fernández \& Hernández, (CALM-1031).

Segunda cita provincial de este taxón que hemos visto con frecuencia a lo largo de la Meseta Cristalina y los Montes de Toledo, en cuyo sector central fue indicada anteriormente (Baonza \& al. 2010). No podemos delimitar con precisión la distribución de nuestro taxón y de la subespecie típica, pero pensamos que el primero debe ser más abundante en la mitad occidental de la provincia.

\section{Myrrhoides nodosa (L.) Cannon}

San Pablo de los Montes, melojares, $1160 \mathrm{msnm}$, 30SUJ8476, 22-V-2010, Hernández Palacios s.n. (CALM-2899).

Umbelífera forestal rara en la provincia de Toledo, donde sólo aparece en la Sierra de San Vicente (Martín-Blanco \& Carrasco 2001, Cantó 2004, García Mateo \& Pajarón 2009) y en localidades oretanas muy cercanas a la nuestra, como ya señalaba Gandoger (1917). Se comporta como especie nemoral en los melojares supramediterráneos en ambos sistemas montañosos. En la distribución peninsular que aporta Jury (2003) se omite la provincia de Toledo.

Nepeta nepetella subsp. aragonensis (Guirao) Aedo

Dos Barrios, coscojares en calizas pontienses, 680 msnm, 30SVK5714, 30-IV-2000, Hernández Palacios s.n. (CALM-2941).

Endemismo de la mitad oriental peninsular, raro en Toledo y enclavado en la Mesa de Ocaña, donde había sido señalada previamente por Laorga (1986), localidad que constituiría su entrada más avanzada hacia el interior en la Submeseta Sur.

Onobrychis matritensis Boiss. \& Reut.

Layos, Sierra de Layos, matorral de Cistus albidus, $770 \mathrm{msnm}$, 30SVK1101, 1-VI-2001, Hernández Palacios s.n. (CALM-2911).

Especie relativamente común en diferentes formaciones de caméfitos del centro de Toledo, principalmente sobre comunidades silicibasófilas de la Meseta Cristalina. Resulta mucho más rara en el Sector Manchego, por lo que territorialmente su comportamiento ecológico no parece incluir las comunidades calcícolas y gipsícolas frecuentes en la Mesa de Ocaña. Se debe añadir esta provincia en la distribución apuntada por Valdés (2000).
Ononis reclinata subsp. mollis (Savi) Bég.

Noblejas, tomillares degradados, $730 \mathrm{msnm}$, 30SVK6126, 27-V-2000, Hernández Palacios s.n. (CALM-942).

Pequeño terófito mediterráneo que localizamos en pastos calcícolas anuales de la Mesa de Ocaña, y que representa la segunda localidad provincial posterior a la mención reciente que hacen Baonza et al. (2010) de los Montes de Toledo, donde constaba una recolección previa (MA-263707).

Ononis viscosa subsp. crotalaroides (Coss.) Sirj.

Guadamur, Camino de Portusa, bordes de olivar, 600 msnm, 30SVK0008, 28-IV-2001, Hernández Palacios s.n. (CALM-2912).

Endemismo del centro y sur de la Península Ibérica. Resulta abundante en arcosas y migmatitas del centro de la provincia, donde puede observarse en comunidades herbáceas subruderales. Las escasas localidades previas conocidas se encuentran referidas también al tramo medio del valle del Tajo (Montserrat 1975, Laorga 1986), en el que encuentra el límite septentrional de su distribución. Sustituiría en tales medios a la subespecie brachycarpa (DC.) Batt. in Batt. \& Trab., taxón iberonorteafricano de carácter basófilo, y que se ha reconocido en el Sector Manchego (Laorga, 1986).

\section{Ornithogalum bourgaeanum Jord.}

San Pablo de los Montes, melojares, $1160 \mathrm{msnm}$, 30SUJ8476, 22-V-2010, Hernández Palacios s.n. (CALM-2910).

No resulta escaso en el sector Toledano-Tagano a la vista de los numerosos testimonios de herbario (MA-355400; MA-355420; MA-465693), aunque faltan citas bibliográficas de poblaciones localizadas. La superposición de caracteres dentro del subgénero Ornithogalum expuesta por Martínez Azorín (2008), que dificulta su estudio, nos hace creer que algunas referencias de $O$. collinum Guss., especie no ibérica, y $O$. baeticum Boiss. podrían corresponder a $O$. bourgaeanum (Baonza et al. 2010, Carrasco 2011, Castillo 1991).

\section{Ornithogalum narbonense L.}

Cobisa, retamares sobre suelos migmatíticos, 660 msnm, 30SVK1107, 5-V-2001, Hernández Palacios s.n. (CALM-2953).

Geófito circunmediterráneo muy extendido en la Península Ibérica. Añadimos esta localidad a las 
que conocíamos (Velasco 1978, Laorga 1986), junto con otra mucho más reciente recogida por Baonza et al. (2010), todas ellas precedidas por otra antigua de Gandoger (1917). Perfilamos así su distribución provincial, que comprende todas las regiones de la mitad oriental desde la Sagra hasta los Montes de Toledo.

Pisum sativus subsp. elatius (M. Bieb.) Asch. \& Graebn.

San Pablo de los Montes, melojares, $1080 \mathrm{msnm}$, 30SUJ8484, 22-V-2010, Hernández Palacios s.n. (CALM-2944); Almorox, encinares, 30TUK7956, 600 msnm, 15-V-2009, Hernández Palacios s.n. (CALM-3048).

Aparece de forma muy puntual en el sotobosque de melojares supramediterráneos en los Montes de Toledo, y también en la Sierra de San Vicente (García Mateo 2003). El material recolectado pertenece a la var. brevipedunculatum P. H. Davis \& Meikle., no conocida hasta la fecha en la provincia de Toledo, y que corresponde al taxón de tendencia más termófila y comportamiento menos ruderal, como señala Romero Zarco (1999).

\section{Polygala rupestris Pourr.}

Dos Barrios, arroyo de la Cañada de la Mora, pastizales en calizas, $680 \mathrm{msnm}$, 30SVK5615, 30-IV2000, Hernández Palacios s.n. (CALM-980).

Pequeño caméfito estenomediterráneo bastante raro dentro de su territorio provincial, el cual comprende únicamente la Mesa de Ocaña, una de sus localidades más interiores en la Península Ibérica. Se ha observado en los escasos afloramientos de conglomerados pliocénicos y rampas pedregosas que coronan los barrancos de la mesa. Conocemos solamente las localidades previas señaladas por Laorga (1986) para esta comarca.

Primula veris subsp. columnae (Ten.) Maire \& Petitmengin

San Pablo de los Montes, melojares sobre cuarcitas, $1000 \mathrm{msnm}$, 30SUJ8470, 22-V-2010, Hernández Palacios s.n. (CALM-2897).

Segunda localidad provincial de este hemicriptófito bastante extendido por el cuadrante nororiental de la Península Ibérica, y que resulta francamente raro en la mitad sur de la misma, donde sólo se conocen poblaciones inconexas en Sierra Morena y la Sierra de Almijara. Nuestra recolección está precedida por otra en Los Yébenes (Baonza et al. 2010). En una y otra localidad también se ha reconocido la subespecie típica (Gómez Manzaneque 1988, Navarro 1996), siendo ambos taxones ignorados en Toledo por Kress (1997).

\section{Ranunculus ophioglossifolius Vill.}

Navalcán, bordes del embalse, comunidades herbáceas de aguas someras, $380 \mathrm{msnm}$, 30TUK1735, 17-IV-2000, Hernández Palacios s.n. (CALM-1032).

Aportamos la segunda cita provincial (Cantó 2004) de este ranúnculo anual muy disperso en la Península Ibérica y característico de comunidades anfibias o acuáticas en aguas oligótrofas; su área provincial comprende las vegas del tramo más occidental del Tajo, Sierra de San Vicente, y extremo oriental de los Montes de Toledo, según el material de herbario visto (MA-648817, MA-680850, MA-338489).

\section{Salvia argentea $\mathrm{L}$.}

Casasbuenas, tomillares en migmatitas, 790 msnm, 30SVK1000, 6-I-2001, Hernández Palacios s.n. (CALM-2895); Urda, bordes de cultivos, 800 msnm, 30SVJ3859, 23-V-2008, Hernández Palacios s.n. (CALM-2894).

Ocasional por toda la provincia en medios subnitrófilos y relativamente eútrofos. Sáenz (2010) considera con dudas su presencia en la misma, pero nos constan varias citas anteriores: Laorga (1986), Egido (1985), Velasco (1978).

\section{Sanguisorba hybrida (L.) Font Quer}

Los Yébenes, Arroyo del Balandrino, quejigares, 860 msnm, 30SVJ1857, 27-V-2005, Hernández Palacios s.n. (CALM-2893).

Aportamos una nueva localización de esta planta endémica de afinidad luso-extremadurense, indicadora de formaciones arbóreas bien conservadas. Se distribuye a lo largo de las vertientes meridionales drenadas a la cuenca del Guadiana en los Montes de Toledo, desde sus tramos orientales a los occidentales, con presencia puntual en la Sierra de San Vicente. La localidad que indicamos aquí constituye, según lo visto, la más oriental de la provincia de Toledo. La localización que indica Gómez Manzaneque (1988), recogida por Sanz Elorza (2006), se sitúa en la provincia de Ciudad Real. Navarro \& Muñoz Garmendia (1998), la señalan de forma dudosa en la provincia. 
Senecio laderoi Pérez-Morales, García-González $\&$ Penas subsp. laderoi

Sonseca, Fuente Techada, juncales nitrificados, 750 msnm, 30SVJ1989, 1-V-2001, Hernández Palacios s.n. (CALM-880).

Se trata del único representante de la sección $D o$ ria (Fabr.) Rchb. en el territorio castellano manchego y el más común por el centro y sur de la Península Ibérica (Pérez Morales et al. 1989), mientras que Senecio doria L. correspondería al taxón de área más amplia (sur y centro de Europa), reconocible por la glabrescencia de sus tallos, que únicamente alcanza el área ibérica en su cuadrante nororiental (Prepirineo, valle del Ebro y sur del Sistema Ibérico Meridional). La indicación aportada por Laorga (1986) para este último taxón, a escasos kilómetros de nuestra localidad y situada en el mismo arroyo, deberá ser comprobada más adelante. Nuestras plantas de Sonseca, tomentosas en todas sus partes, fueron herborizadas en comunidades de Scirpoides holoschoenus (L.) Soják de escaso interés y acompañadas de otras especies nitrófilas, sobre suelos oligótrofos de textura gruesa.

\section{Sideritis paulii $\mathrm{Pau}$}

Los Yébenes, La Morra, claros de encinar, 800 msnm, 30SVJ2765, 27-V-2005, Hernández Palacios s.n. (CALM-2946).

Caméfito endémico del sudoeste peninsular del que aportamos otra localidad en las sierras más orientales de los Montes de Toledo que amplia una nueva cuadrícula de 10 kilómetros de lado su área conocida. Las plantas inicialmente identificadas como $S$. hirsuta L. por Gómez Manzaneque (1988) en la localidad cercana de Quintos de Mora, sirvieron más tarde para describir $S$. calduchii Cirujano, R. Roselló, Peris \& Stübing, endemismo estrictamente oretano y cuyo areal comprendería toda esta alineación orográfica, desde la provincia de Cáceres hasta el municipio de Los Yébenes (Cirujano et al. 1994). Más recientemente Morales (2010) ha incluido este último taxón dentro de las formas de variación de $S$. paulii. No obstante, el examen de los caracteres ofrecidos por el indumento permitiría adscribir nuestras plantas de Los Yébenes a $S$. calduchii. Todos los taxones de la subsección Linariifolia se encuentran recogidos en el Catálogo Regional de Especies Amenazadas, bajo la categoría de Interés Especial (Decreto 200/2001, de 6 de noviembre, por los que se crea y modifica el Catálogo Regional de Especies
Amenazadas).

\section{Silene almolae J. Gay}

Noblejas, salviares en calizas, $730 \mathrm{msnm}$, 30SVK6028, 27-V-2000, Hernández Palacios s.n. (CALM-938).

Endemismo ibérico meridional. Sanz Elorza (2006) recoge una mención genérica en la provincia, y otra aportada por Laorga (1986), ninguna de ellas respaldada por testimonios de herbario, por lo que la nuestra constituiría la primera cita localizada en Toledo. Las recolecciones en territorios próximos de la provincia de Madrid son sin embargo numerosas, en contraste con su rareza en el tramo toledano de la fosa del Tajo. Es taxón de marcada afinidad ibero-levantina, y en las localidades estudiadas hemos encontrado un número muy reducido de ejemplares.

\section{Silene laeta Aiton}

Velada, El Baldío, arenales, $400 \mathrm{msnm}$, 30TUK3033, 13-V-2008, Hernández Palacios s.n. (CALM-885).

Hemicriptófito de amplia distribución general (áreas mediterráneas y eurosiberiana occidentales), distribuida por la mitad occidental de la Península Ibérica, en la que rehúye sin embargo los territorios más continentales. En la localidad visitada crecía de forma muy abundante en compañía de numerosas especies anfibias y helófitas. Talavera (1990) señala su presencia en la provincia, pero no hemos encontrado otras citas en la bibliografía ni material de herbario.

\section{Stipa bromoides (L.) Dörfler}

Urda, encinares incipientes en cuarcitas, 1030 msnm, 30SVJ4257, 24-V-2008, Hernández Palacios s.n.(CALM-2945).

Especie circunmediterránea muy rara en la provincia. Ecológicamente es una planta indiferente edáfica que, en la zona central de la península ibérica, habita bosques mesomediterráneos templados en estado de recuperación más o menos avanzado. La única mención que hemos encontrado en la provincia de Toledo es la que recogen Vázquez \& Devesa (1996) a partir de material recolectado por P. Montserrat en "Santa Quiteria", que asimilamos a la localidad de Minas de Santa Quiteria, alquería perteneciente al municipio de Sevilleja de la Jara. Entre una y otra localidad, distantes casi $120 \mathrm{~km}$, no descartamos la existencia de otras po- 
blaciones en la vertiente septentrional de los montes oretanos.

\section{Trifolium stellatum L.}

San Pablo de los Montes, melojares, $1080 \mathrm{msnm}$, 30SUJ8476, 22-V-2010, Hernández Palacios s.n. (CALM-2947); Caleruela, dehesas, $500 \mathrm{msnm}$, 30SUK0815, 5-V-2008, Hernández Palacios s.n. (CALM-2948); Almorox, encinar aclarado, 540 msnm, 30TUK3855, 12-V-2009, Hernández Palacios s.n. (CALM-2949).

Común en los Montes de Toledo, Sierra de San Vicente y a lo largo del tramo inferior del río Tajo, en rangos de altitud que comprenden los pisos más basales hasta cotas del piso supramediterráneo. En la distribución provincial indicada por Muñoz Rodríguez et al. (2000) se considera como dudosa en Toledo, pero las citas previas son numerosas: Laorga (1986) y Egido (1985) en la Meseta Cristalina, Velasco (1978) y Baonza et al. (2010) en los Montes de Toledo, y García Mateo (2003) en la Sierra de San Vicente.

Trifolium sylvaticum Gérard ex Loisel.

Hinojosa de San Vicente, encinares, $640 \mathrm{msnm}$, 30TUK5341, 11-V-2002, Hernández Palacios s.n. (CALM-915).

Trébol anual característico de pastizales silicícolas de terófitos, no muy común en la provincia. Laorga (1986) la indicaba (sub Trifolium smyrnaeum Boiss.) en suelos arenosos de Carranque. La cita aquí indicada constituye la segunda localidad en Toledo. La distribución peninsular es amplia, pero sus exigencias bioclimáticas de una moderada continentalidad podrían explicar la escasez de poblaciones observada.

\section{Trigonella gladiata Steven}

Villarrubia de Santiago, aljezares, $660 \mathrm{msnm}$, 30SVK7226, 30-IV-2010, Hernández Palacios s.n. (CALM-2940).

Especie mediterránea de distribución peninsular ibero-levantina. Aportamos la segunda localidad conocida en la provincia. Anteriormente, Laorga

(1986) la mencionaba únicamente en majadales calcícolas de la localidad de Borox, fuera de la Mesa de Ocaña, donde la hemos herborizado nosotros.

Trisetum loeflingianum (L.) C. Presl

Yepes, salviares ralos en calizas, $600 \mathrm{msnm}$,
30SVK3821, 5-V-2009, Hernández Palacios s.n. (CALM-2939).

Nanoterófito mediterráneo de área ibérica oriental que se encuentra francamente extendido en la Mesa de Ocaña y por los terrenos miocenos de la Cuenca del Tajo. Laorga (1986) indica una sola localidad situada en el entorno próximo de la nuestra, sin que conozcamos menciones de otros autores. Fue observada tanto en suelos calizos como yesíferos.

\section{Veronica verna $\mathrm{L}$.}

Pulgar, Cerro del Gango, pastos anuales en migmatitas, 800 msnm, 30SVJ0096, 20-IV-2001, Hernández Palacios s.n. (CALM-2956); Mazarambroz, retamares, $610 \mathrm{msnm}$, 30SVJ0699, 21IV-2001, Hernández Palacios s.n. (CALM-2955).

No conocida anteriormente en los territorios silicibasófilos de la Meseta Cristalina, donde no es sin embargo escasa. Con el material de herbario estudiado y las muy escasas citas previas trazamos su distribución provincial: áreas toledano-taganas meridionales y Sierra de San Vicente (Cantó, 2004; Martín Blanco \& Carrasco, 2001; Baonza et al. 2010).

\section{Viola canina L.}

San Pablo de los Montes, melojares, $1050 \mathrm{msnm}$, 30SUJ8476, 22-V-2010, Hernández Palacios s.n. (CALM-2952).

Nuestra recolección está precedida únicamente por la de Baonza et al. (2010). Fue observada en ambientes nemorales del tramo central de las alineaciones oretanas. No obstante, son numerosas las poblaciones también oretanas diseminadas en el territorio contiguo de Ciudad Real (MartínBlanco \& Carrasco 2005). En esta localidad conviviría además con Viola suavis M. Bieb. (Velasco, 1978; MA-508647), otra violeta perenne de la sección Viola de área peninsular oriental sin embargo, y sin embargo bastante rara en las provincias más interiores.

\section{Agradecimientos}

Al Dr. Santiago Pajarón, que continuando el apoyo que nos ofrece en nuestra labor botánica desde hace tiempo, ha mejorado el manuscrito con interesantes observaciones.

A la Dra. Charo Noya, por la ayuda prestada en el estudio de los materiales del herbario MA y 
demás personal del mismo. Por las mismas razones y sus amistosos consejos, al Dr. José Pizarro, Conservador del herbario MAF.

\section{Referencias}

Arán VJ. 1995: Fragmenta Chorologica Occidentalia, 5681-5692. Anales del Jardín Botánico de Madrid 53(2): 243-244.

Baonza Díaz J, Caparrós Callejo R, García Medina N, Martínez García F \& Gómez Manzaneque F. 2010: Flora vascular de los Quintos de Mora (Los Yébenes, Toledo). Ecología 14: 39-58.

Benedí C, Molero J, Simon J \& Vicens J. 1997: Euphorbia L. In Flora Iberica, Vol. VIII, HaloragaceaeEuphorbiaceae (Castroviejo S. et al. eds.). Madrid: Real Jardín Botánico de Madrid, CSIC, pp. 210-285.

Boissier, P.E. 1839: Voyage botanique dans le midi de L'Espagne pendant l'anne 1837. Vol. II, Paris, Gide et Cie., Libraires-éditeurs.

Cantó P. 1979: Estudio de las comunidades arbóreas, arbustivas y esciófilas de la Sierra de San Vicente (Toledo). Madrid: Facultad de Farmacia. Universidad Complutense de Madrid. Tesis de Licenciatura.

Cantó P. 2004: Estudio fitosociológico y biogeográfico de la sierra de San Vicente y tramo inferior del valle del Alberche (Toledo, España). Lazaroa 25: $187-$ 249.

Carrasco MA. 2011: Notas de flora hispánica, VII. Botanica Complutensis 35: 89-90.

Castillo Gorruño JL. 1991: Contribución al estudio de la flora y vegetación vascular de la cuenca del río Sangrera (Toledo, España). Madrid: Facultad de Biología. Universidad Complutense de Madrid.Tesis de Licenciatura.

Castillo JL \& Carrasco MA. 1992: Fragmenta Chorologica Occidentalia, 4180-4189. Anales del Jardín Botánico de Madrid 50(1): 100.

Chaytor DA \& Turrill WB. 1935: The genus Clypeola and its intraspecific variation. Bulletin of Miscellaneous Information 1: 1-24

Cirujano S, Roselló R, Peris JB \& Stübing G. 1994: Sideritis calduchii, sp. nov. (Labiatae), endemismo ibérico. Anales del Jardín Botánico de Madrid 52(1): 109-111.

Egido P. 1985: Contribución al conocimiento de la flórula toledana (Toledo, Polán y la Puebla de Montalbán). Toledo: Instituto Provincial de Investigaciones y Estudios Toledanos. Diputación de Toledo.

Fernández González F, Pérez Badia R, Bouso V, Crespo G, Rodríguez Rojo MP, Rodríguez Torres A, Rojo J \& Sardinero S. 2012: Síntesis de la vegetación de la provincia de Toledo. In Avances en el conocimiento de la vegetación. XXIII Jornadas Internacionales de Fitosociología. Libro de Actas. Ediciones de la Universidad de Castilla-La Mancha, pp. 97-160.

Gandoger M. 1917: Catalogue des plantees récoltées en Espagne et Portugal. Paris.

García Mateo R. 2003: Flora vascular de la sierra de
San Vicente (Toledo, España). Madrid: Facultad de Biología. Universidad Complutense de Madrid.Tesis de Licenciatura.

García Mateo R \& Pajarón S. 2009: Flora y vegetación de la Sierra de San Vicente. Toledo. Servicio de Publicaciones de la Excma. Diputación de Toledo.

Gómez Manzaneque M. 1988: La cubierta vegetal de los Montes de Mora (Los Yébenes, Toledo). Ecología 2:111-130.

Izco J. 1974: Pastizales terofíticos de la provincia de Madrid. Thero-Brachypodion y Sedo-Ctenopsion. Anales Instituto Botánico A.J. Cavanilles 31(1): 209224.

Jiménez Mejías P \& Luceño M. 2007: Eleocharis L. In Flora Iberica, Vol. XVIII, Cyperaceae-Pontederiaceae (Castroviejo S. et al. eds.). Madrid: Real Jardín Botánico de Madrid, CSIC, pp. 75-91.

Jury SL. 2003: Myrrohoides Heist. In Flora Iberica, Vol. $X$, Araliaceae-Umbelliferae (Nieto Feliner G. et al. eds.). Madrid: Real Jardín Botánico de Madrid, CSIC, pp. 63-65.

Kress A. 1997: Primula L. In Flora Iberica, Vol. V, Ebenaceae-Saxifragaceae (Castroviejo $\mathrm{S}$. et al. eds.). Madrid: Real Jardín Botánico de Madrid, CSIC, pp. 10-22.

Ladero Alvarez msnm, Navarro Andrés F, Valle Gutiérrez CJ, Pérez Chiscano JL, Santos Bobillo MT, Ruiz Téllez MT, Fdez.-Arias MI, Valdés Franzi A \& González FJ. 1985: Comunidades herbáceas de lindero, en los bosques carpetano-iberico-leoneses y luso-extremadurenses. Studia botanica 4: 7-26.

Laorga S. 1986: Estudio de la flora y vegetación de las comarcas toledanas del tramo central de la cuenca del Tajo. Madrid: Facultad de Farmacia. Universidad Complutense de Madrid.Tesis Doctoral.

Marcos N. 1985: Flora y vegetación de la comarca de Puerto Lápice y sierra de Herencia. Madrid: Facultad de Biología. Universidad Complutense de Madrid.Tesis Doctoral.

Martín-Blanco CJ \& Carrasco MA. 2001. Notas de flora hispánica, IV. Botanica Complutensis 25: 309-312.

Martín-Blanco CJ \& Carrasco MA. 2005: Catálogo de la flora vascular de la provincia de Ciudad Real. Madrid: Monografías de la Asociación de Herbarios Ibero-Macaronésicos.

Martínez Azorín M. 2008: Sistemática del género Ornithogalum L. (Hyacinthaceae) en el Mediterráneo Occidental: implicaciones taxonómicas, filogenéticas y biogeográficas. Alicante: Departamento de Ciencias Ambientales y Recursos Naturales. Universidad de Alicante.Tesis Doctoral.

Mateo Sanz G \& Arán Redó VJ. 2002: Nuevos datos sobre flora de la provincia de Cuenca (XVII). Flora Montiberica 20: 1-5.

Molero Briones J \& Montserrat i Marti JM. 1983: Contribución al conocimiento de la flora del Sistema Ibérico septentrional. Collectanea Botanica 14: 347374.

Montserrat P. 1975: Notas sobre flora española. Acta Botanica Malacitana 1: 43-75.

Montserrat P \& Hernández Cardona AM.1983: Avena eriantha Durieu nueva para la flora ibérica. Anales 
del Jardín Botánico de Madrid 39(2): 546.

Morales R. 1997: El género Calamintha Mill. (Labiatae) en la Península Ibérica e Islas Baleares. Anales Jard. Bot. Madrid 55(2): 261-276.

Morales R. 2010: Calamintha L. In Flora Iberica, Vol. XII, Verbenaceae-Labiatae-Callitrichaceae (Morales R. et al.eds.). Madrid: Real Jardín Botánico de Madrid, CSIC, pp. 431-435.

Morales R. 2010: Sideritis L. In Flora Iberica, Vol. XII, Verbenaceae-Labiatae-Callitrichaceae (Morales R. et al.eds.). Madrid: Real Jardín Botánico de Madrid, CSIC, pp. 234-288.

Muñoz Rodríguez A, Devesa JA, Talavera S. 2000: Trifolium L. In Flora Iberica, Vol. VII(II), Leguminosae (p.p.) (Talavera S. et al.eds.). Madrid: Real Jardín Botánico de Madrid, CSIC, pp. 647-719.

Navarro C \& Muñoz Garmendia F. 1998: Sanguisorba L. In Flora Iberica, Vol. VI, Rosaceae (Muñoz Garmendia F \& Navarro C. eds.). Madrid: Real Jardín Botánico de Madrid, CSIC, pp. 375-388.

Navarro García P. 1996: Estudio de la flora vascular de la Sierra de San Pablo de los Montes (Toledo). Madrid: Facultad de Ciencias Biológicas. Universidad Complutense de Madrid.Tesis de Licenciatura.

Neves S. 2003: Bupleurum L. In Flora Iberica, Vol. X, Araliaceae-Umbelliferae (Nieto Feliner G. et al. eds.). Madrid: Real Jardín Botánico de Madrid, CSIC, pp. 240-265.

Nogueira i \& Paiva J. 1996: Malva L. In Flora Iberica, Vol. III, Plumbaginaceae (p.p.)-Capparaceae (Castroviejo S. et al. eds.). Madrid: Real Jardín Botánico de Madrid, CSIC, pp. 208-266.

Pérez Morales C, García González ME \& Penas Merino A. 1989: Revisión taxonómica de las especies ibéricas de la sección Doria (Fabr.) Reichenb. del género Senecio L. Studia botanica: 117-127.

Podlech D. 2000: Astragalus L. In Flora Iberica, Vol. VII(II), Leguminosae (p.p.) (Talavera S. et al. eds.). Madrid: Real Jardín Botánico de Madrid, CSIC, pp. 279-338.

Rivas Goday S \& Rivas Martínez S. 1969: Matorrales y tomillares de la Península Ibérica comprendidos en la clase Ononido-Rosmarinetea Br.-BI. 1947. Anales del Instituto Botánico A. J. Cavanilles 25: 5-197.

Rivas Goday S, Borja Carbonell J, Esteve Chueca F, Fernández-Galiano E, Mansanet $\mathrm{J}$, Monasterio Fernández A \& Ocaña M. 1959: Aportaciones a la Fitosociología hispánica (Proyectos de comunidades hispánicas). Nota III. Anales del Instituto Botánico A. J. Cavanilles 16: 465-472.

Romero Zarco C. 1984: Revisión taxonómica del género Avenula (Dumort.) Dumort. en la Península Ibérica e Islas Baleares. Lagascalia 13(1): 39-146.

Romero Zarco C. 1990: Las avenas del grupo Barbata en la Península Ibérica y Baleares. Lagascalia 16(2): 243-268

Romero Zarco C. 1993: Observaciones sobre las
Avenula del grupo Marginata en Andalucía. Acta Botanica Malacitana 18: 147-151.

Romero Zarco C. 1999: Pisum L. In Flora Iberica, Vol. VII(I), Leguminosae (p.p.) (Talavera S. et al. eds.). Madrid: Real Jardín Botánico de Madrid, CSIC, pp. 481-486.

Romero Zarco C. 1999: Vicia L. In Flora Iberica, Vol. VII(I), Leguminosae (p.p.) (Talavera S. et al. eds.). Madrid: Real Jardín Botánico de Madrid, CSIC, pp. 360-417.

Romero Zarco C. 2009: Avena L. in Flora Vascular de Andalucía Oriental, Vol. 1, Selaginellaceae-Ceratophyllaceae (Talavera, S. et al. eds.). Sevilla: Consejería de Medio Ambiente, Junta de Andalucía, pp. 321-323.

Romero Zarco C. 2010: Juncus L. In Flora Iberica, Vol. XVII, Butomaceae-Juncaceae (Talavera S. et al. eds.). Madrid: Real Jardín Botánico de Madrid, CSIC, pp. 123-186.

Sáenz, L., 2010: Salvia L. In Flora Iberica, Vol. XII, Verbenaceae-Labiatae-Callitrichaceae (Morales R. et al.eds.). Madrid: Real Jardín Botánico de Madrid, CSIC, pp. 298-325.

Sales F \& Hedge, IC. 1999: Medicago L. In Flora Iberica, Vol. VII(I), Leguminosae (p.p.) (Talavera S. et al. eds.). Madrid: Real Jardín Botánico de Madrid, CSIC, pp. 741-775.

Sanz Elorza E. 2006: Aproximación al catálogo florístico de la provincia de Toledo. Ecología 20: 89-162.

Segura Zubizarreta A. 1975: De flora soriana y otras notas botánicas. Anales del Instituto Botánico A. J. Cavanilles 32(2): 763-774.

Simon i Pallisé J. 1993: Estudis biosistemàtics en Euphorbia L. subsect. Galarrhaei (Boiss.) Pax (grup d'E. flavicoma i espècies afins) a la Mediterrània occidental. Barcelona: Facultad de Farmacia. Universidad de Barcelona.Tesis Doctoral.

Talavera S. 1990: Silene L. In Flora Iberica, Vol. II, Platanaceae-Plumbaginaceae (p.p.) (Castroviejo S. et al. eds.). Madrid: Real Jardín Botánico de Madrid, CSIC, pp. 313-406.

Valdés B. 2000: Onobrychis Mill. In Flora Iberica, Vol. VII(II), Leguminosae (p.p.) (Talavera S. et al. eds.). Madrid: Real Jardín Botánico de Madrid, CSIC, pp. 955-970.

Vaquero de la Cruz J. 1992: Flora del Parque Natural de Cabañeros (Montes de Toledo, Ciudad Real). Ecología 7: 79-111.

Vázquez FM \& Devesa JA. 1996: Revisión del género Stipa L. y Nasella Desv. (Poaceae) en la Península Ibérica e Islas Baleares. Acta Botanica Malacitana 21: 125-189.

Velasco A. 1978: Contribución al estudio de la flora y vegetación de la Comarca granítica toledana y Montes de Toledo (tramo oriental). Madrid: Facultad de Farmacia. Universidad Complutense de Madrid.Tesis Doctoral 